\title{
Fotovoz, una metodología de investigación acción participativa, aplicada para evaluar una actividad académica en una materia de Grado en Podología
}

\author{
Coronado Carvajal, Carmen; Freijomil-Vázquez, Carla²; Fernández-Basanta, Sara3; \\ Andina-Díaz, Elena ${ }^{4}$; Movilla-Fernández, María-Jesús ${ }^{5}$ \\ 1,2,3,5 Grupo de investigación GRINCAR, GID-Enfermería-UDC, Facultad de Enfermería y \\ Podología, Universidade da Coruña, \\ ${ }^{4}$ Grupo de investigación SALBIS, Facultad de Ciencias de la Salud, Universidad de León; \\ Grupo de investigación Enfermería y Cultura del Cuidado (EYCC), \\ Facultad de Ciencias de la Salud, Universidad de Alicante \\ Códigos ORCID: '0000-0002-4824-6902, ²0000-0003-3961-1899, \\ ${ }^{3} 0000-0002-7542-9667,{ }^{4} 0000-0001-9687-1967,{ }^{5} 0000-0001-9369-8636$
}

\section{RESUMEN}

Entre las actividades académicas de la asignatura "Microbiología y Parasitología" de Grado en Podología se incluyó una visita guiada a la depuradora de aguas residuales de A Coruña. El objetivo de este trabajo es presentar una metodología de investigación acción participativa para evaluar la pertinencia de la actividad docente. Se ha implementado el uso de diferentes pasos de fotovoz que fomenta la participación de la comunidad estudiantil a través de la fotografía y facilita la reflexión crítica sobre una realidad concreta, con el objetivo final de ser colectivamente conscientes. Cuarenta y tres estudiantes universitarios de edad comprendida entre los 18 y 45 años (edad media 22,9) presentaron foto en color y reflexiones sobre ella en su informe individual. Los resultados se obtuvieron del análisis cualitativo de las fotografías, las narraciones del informe, y la transcripción del grupo de discusión posterior realizado en clase. Se identificaron ocho categorías principales que emergieron de los datos. Consideramos muy positiva la aplicación de esta metodología cualitativa para complementar actividades formativas que se realizan en 
entorno real y que implican acciones con consecuencias para la comunidad. Fotovoz se puede considerar eficaz para introducir contenido transversal de sostenibilidad en los currículos de campus verdes.

PALABRAS CLAVE: estudiantes universitarios; fotovoz, visita guiada; investigación cualitativa

\section{CITA RECOMENDADA:}

Coronado Carvajal, Carmen; Freijomil-Vázquez, Carla; Fernández-Basanta, Sara; Andina-Díaz, Elena; Movilla-Fernández, María-Jesús (2020): Fotov0z, una metodología de investigación acción participativa, aplicada para evaluar una actividad académica en una materia de Grado en Podología. En De la Torre Fernández, E. (ed.) (2020). Contextos universitarios transformadores: Boas prácticas no marco dos GID. IV Xornadas de Innovación Docente. Cufie. Universidade da Coruña. A Coruña (págs. 315-328). DOI capítulo: https://doi.org/10.17979/spudc.9788497497756.315

DOI libro: https://doi.org/10.17979/spudc.9788497497756

\section{ABSTRACT}

Among the academic activities of the subject "Microbiology and Parasitology" of Degree in Podiatry, a guided visit to the wastewater treatment plant of A Coruna was included. The objective of this work is to present a participatory action research methodology to evaluate the relevance of the above teaching activity. The use of different photovoice steps was implemented. Photovoice encourages the participation of the student community through photography and facilitates critical reflection on a specific reality, with the ultimate goal of being collectively aware. Forty-three university students aged between 18 and 45 years (average age 22.9) presented a color photo and reflections on it in their individual report. The results were obtained from the qualitative analysis of the photographs, the narratives of the report, and the transcription of the subsequent discussion group conducted in class. Eight main categories that emerged from the data were identified. We consider the application of this qualitative methodology to complement teaching assignments very positive. Especially, for those activities that are carried out in a real environment and that involve actions with consequences for the community. Photovoice can be considered effective in introducing transversal sustainability content in green campus curricula.

KEY WORDS: university students; photovoice; guided visit; qualitative research 


\section{INTRODUCCIÓN}

La fotografía participativa o fotovoz se define como una metodología cualitativa de investigación acción participativa que tiene la particularidad de permitir a las personas narrar sus experiencias y producir conocimiento sobre el contexto en el que viven (Wang, 2003). Wang and Burris (1997) establecieron las siguientes características de esta metodología de investigación cualitativa:

- mediante las fotografías permite a los participantes mostrar su punto de vista sobre sus ideas, preocupaciones y miradas a los problemas que les conciernen

- las fotografías se convierten en objetos potentes para el análisis y la construcción de conocimiento mediante estrategias cualitativas

- fotovoz es una estrategia de difusión de propuestas para que lleguen a aquellas personas con poder de decisión para promover cambios.

La metodología fotovoz se desarrolló a mediados de los años noventa por Caroline Wang y colaboradores (se apoyaron para ello en la Teoría "Critical consciousness" de P. Freire y en teorías feministas), y se ha utilizado principalmente como un método de investigación para problemas de Salud Pública y Justicia Social.

El abanico de temas investigado con fotovoz abarca desde enfermedades infecciosas epidémicas y problemas crónicos de salud hasta temas de violencia y discriminación. Igualmente se ha utilizado con diferentes grupos de edad y en países diversos (Catalani \& Minkler, 2010).

Sin embargo, en la literatura más reciente se ha demostrado su valor como herramienta pedagógica, particularmente atractiva para estudiantes universitarios (Edwards et al., 2012; Lichty, 2013). Concretamente en el campo de los estudiantes de grado en titulaciones sanitarias la metodología fotovoz se ha usado, no como un método de investigación, sino como una estrategia de innovación docente para fortalecer la conexión entre lo didáctico y lo clínico. Por ejemplo, en la materia de Enfermería comunitaria de la titulación de Grado en Enfermería, el uso de fotovoz permitió a los estudiantes desarrollar un sentido sobresaliente 
para priorizar las intervenciones comunitarias cuando los estudiantes fueron capaces de integrar la voz de la comunidad para desarrollar estas intervenciones. El uso de las fotografías para pensar sobre las necesidades y fortalezas de la comunidad alentó a los estudiantes a crear un diálogo entre ellos, el profesorado y los miembros de la comunidad sobre posibles soluciones. (Fernandes, Ferreira, \& Marques, 2018; Gallagher \& Stevens, 2015; Leipert \& Anderson, 2012).

El alumnado del primer curso académico de la titulación de Grado en Podología de la Universidade da Coruña debe cursar en el segundo cuatrimestre la asignatura "Microbiología y Parasitología". Los contenidos del Bloque temático de Microbiología ambiental de esta asignatura están relacionados con la contaminación microbiana de las aguas recreativas naturales y artificiales, como fuentes de infección para el pie. Este contenido es importante para los futuros profesionales podólogos.

Desde hace algunos cursos académicos, se ha incluido una actividad académica entre las actividades docentes de esta asignatura que consiste en una visita guiada a la depuradora de aguas residuales de A Coruña.

Los objetivos docentes de la actividad son:

- reforzar los contenidos microbiológicos a través de la observación, e

- introducir de manera transversal la conciencia ciudadana sobre la sostenibilidad y el uso responsable del agua.

Este artículo presenta los resultados del uso de diferentes pasos de la metodología fotovoz como una metodología cualitativa de acción participación que pretende provocar la participación de la comunidad estudiantil a través de la fotografía y la reflexión crítica sobre una realidad concreta, el uso correcto del agua como bien de la comunidad, con el objetivo final de ser colectivamente conscientes. Esta investigación ha implementado el método de investigación cualitativa fotovoz para evaluar una actividad académica realizada en entorno real fuera del aula, y ha explorado el impacto en los estudiantes universitarios de la observación "in 
situ" de acciones de sostenibilidad ambiental para la recuperación de aguas residuales industriales y comunitarias desarrolladas por la empresa municipal de la ciudad.

\section{DESCRIPCIÓN DE LA EXPERIENCIA}

En este estudio, el alumnado de primer curso de la titulación de Grado en Podología ha fotografiado, discutido y participado en un análisis sobre el uso correcto del agua y las necesidades de depuración del agua usada tanto por la comunidad a la que pertenecen como por las industrias del entorno.

La experiencia fotovoz se ha desarrollado en la Facultad de Enfermería y Podología de la Universidade da Coruña, situada en el Campus de Ferrol.

El Campus de Ferrol participa en el Programa de Green Campus de la Universidade da Coruña desde 2017. En octubre de 2019 ha obtenido el certificado y la bandera verde del programa Eco-Escuelas de la Asociación para la Defensa del Consumidor (ADEAC) que está asociada a la Fundación para la Educación Ambiental (FEE), siendo el segundo Campus completo, a nivel estatal y autonómico, en obtenerlo. Estos distintivos reconocen los esfuerzos realizados por la comunidad universitaria trabajando hacia un estilo de vida sostenible. En este contexto, la preocupación por el medio ambiente se hace patente en el ámbito docente, se han incluido en las guías docentes una serie de recomendaciones "Green Campus", y se ha sugerido la inclusión de competencias transversales.

En este pequeño campus, en el noroeste España, estudiaron 1.957 jóvenes universitarios durante el curso académico 2018-2019 (fuente UDC). La población estudiantil en la Facultad de Enfermería y Podología estaba formada por 459 estudiantes, de los cuales 94 eran hombres y 365 mujeres. En la asignatura "Microbiología y parasitología" se matricularon 55 estudiantes.

La visita guiada a la planta de tratamiento de aguas residuales de A Coruña (EDAR Bens, A Coruña, España) se ofertó a todos los estudiantes matriculados en "Microbiología y Parasitología" como una de las actividades académicas de la asignatura. Esta empresa 
supramunicipal de la provincia de A Coruña ofrece regularmente una visita informativa guiada dirigida a estudiantes y ciudadanos de la zona. La profesora responsable de la asignatura (primera autora) solicitó dos visitas para el mes de mayo coincidiendo con el trabajo en clase de la unidad temática dedicada a Microbiología del agua. La asistencia a la visita y la entrega del informe individual contribuyeron en la evaluación del estudiante con 0,5 puntos de la calificación final. Cinco estudiantes no pudieron realizar la actividad porque la visita no es recomendable para personas asmáticas, dado que los fuertes olores de la planta pueden desencadenar una crisis asmática. Este alumnado estaba exento de realizar la actividad debido a razones médicas. Tres estudiantes matriculados nunca han asistido a clase, y los tres estudiantes repetidores no quisieron participar en la actividad puesto que ya la habían realizado el año anterior y se les mantenían los 0,5 puntos en su evaluación continua. Los estudiantes restantes se dividieron en dos grupos para asistir a la visita el 9 (23 personas), y el 16 de mayo (21 personas).

La participación de los estudiantes en la experiencia fotovoz se propuso de forma voluntaria y la no participación no significaría penalización alguna en la nota final de la materia. Se pidió a los estudiantes voluntarios que incluyesen en el informe individual sobre la visita:

- la fotografía,

- un documento explicativo de la misma, y

- el consentimiento para participar en la experiencia.

Solo un estudiante no incluyó una fotografía de fotovoz en el informe individual. Finalmente, 43 estudiantes participaron en la experiencia docente innovadora. 


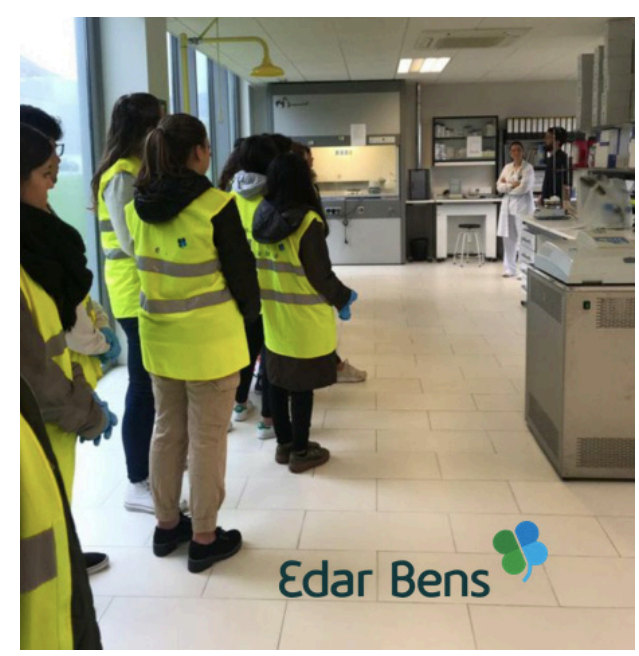

Figura 1: Grupo de alumnos de Podología visitando el laboratorio de la planta depuradora

Esta experiencia fotovoz se presentó en clase, en una sesión de grupo completo, antes de la fecha de las visitas, a todos los estudiantes matriculados. En esta reunión inicial del grupo, se explicaron los objetivos y el alcance de la experiencia, y el modelo de informe que debían completar si decidían participar en fotovoz. El modelo de informe incluía una página para enviar la fotografía y el método mnemotécnico SHOWED. El documento SHOWED está indicado para guiar las narraciones sobre por qué cada participante tomó esa fotografía y lo que significaba para el/la autor/a (Wallerstein \& Bernstein, 1988; Wang \& Burris, 1997). El documento incluye una línea para el título de la fotografía y 5 preguntas (Catalani \& Minkler, 2010; Wallerstein \& Bernstein, 1988):

- $\quad \mathrm{S}(\mathrm{See})$ ¿Qué ves aquí?

- H (Happening) ¿Qué está pasando realmente?

- 0 (Our lives) ¿Cómo se relaciona esto con nuestras vidas?

- W (Why) ¿Por qué existe este problema?

- E (Educate) ¿Podemos usar esta foto para educar?

- $\quad \mathrm{D}(\mathrm{Do})$ ¿Qué podemos hacer al respecto? 
El grupo recibió instrucciones sobre cómo obtener una fotografía que no debe identificar personas o lugares, debe respetar el anonimato de los participantes y no debe ser ofensiva 0 degradante para ninguna persona. Se pidió que se incluyera un documento de consentimiento firmado para el uso de las fotos con fines de enseñanza e investigación.

En el modelo de informe, se invitó a los estudiantes a tomar fotografías sobre una de las siguientes tres preguntas:

- ¿Hacemos un uso responsable del agua?

- ¿Ha influido de alguna manera en mi vida diaria el conocimiento sobre el proceso de purificación del agua?

- ¿Qué puedo cambiar en mi vida diaria con respecto al uso del agua para contribuir a la sostenibilidad ambiental?

Para garantizar el anonimato, los nombres de los estudiantes participantes se codificaron asignando la letra $E$ (Estudiante) seguida de un número ordinario. Después de un análisis preliminar de los 43 informes individuales se invitó a dieciséis estudiantes para participar en un grupo de discusión. Esta sesión grupal se desarrolló en una reunión final con presencia de todos los estudiantes de la clase. Dos facilitadoras utilizaron una presentación de ordenador en la pantalla, con las 16 fotografías elegidas y el método SHOWED para guiar la discusión. La función de las facilitadoras fue involucrar a los participantes y asegurarse de que cada participante pudiera explicar su fotografía. Finalmente, se agradeció a todos los estudiantes la participación en la actividad. Se anunció la publicación de las fotos seleccionadas en el tablón de anuncios Green Campus Ferrol de la Facultad. El grupo de discusión se grabó en audio digitalmente y se transcribió posteriormente.

Los datos cualitativos recopilados de los informes de los estudiantes y la transcripción del grupo de discusión se analizaron manualmente. Se realizó un análisis de contenido temático. Se buscaron, codificaron y agruparon unidades significativas en las categorías principales. Las categorías se discutieron entre los investigadores y los estudiantes, para validar los datos. Los 
datos obtenidos en las fotografías ayudaron a triangular la información. Se mantuvo una actitud de autocrítica durante todo el proceso, para lograr la reflexividad.

\section{RESULTADOS}

Cuarenta y tres estudiantes universitarios del número total de estudiantes de podología de primer año inscritos en la asignatura "Microbiología y Parasitología" presentaron 43 fotos en color y 43 reflexiones en sus informes individuales de la visita a la planta de tratamiento de aguas residuales.

La edad de los participantes estaba comprendida entre los 18 y 45 años (edad media 22,9), 37 de ellos eran mujeres.

Los resultados se obtuvieron del análisis cualitativo de las fotografías, las narraciones correspondientes basadas en SHOWED y la transcripción del grupo de discusión.

Se identificaron ocho categorías principales que emergen de las fotografías y sus narrativas. Estas categorías principales fueron:

- (1) ahorro / desperdicio de agua,

- (2) mal uso del WC,

- (3) eliminación de aceite usado,

- (4) desechos sólidos / basura,

- (5) reutilización de agua limpia,

- (6) reutilización / reducción del uso de plásticos,

- (7) vida acuática, y

- (8) necesidades de educación y concienciación en sostenibilidad en la comunidad y las empresas.

En la Figura 2 se muestran los pósteres que el Grupo de Innovación Docente (GID_Enfermería_UDC) diseñó para la difusión de los resultados de esta experiencia en el Tablón Green Campus de la Facultad de Enfermería y Podología. 
El póster de la Figura 2A, recoge fotografías correspondientes a cuatro de las categorías que emergieron de los resultados del análisis cualitativo donde se critican 0 afean comportamientos relacionados con el mal uso del agua en actividades relacionadas con el hogar como la cocina, limpieza, o la higiene personal. Igualmente aparece el problema de la basura que se acumula en nuestras calles y que acaba contaminando todas las aguas residuales ya que en nuestro medio se combinan en la misma planta depuradora las aguas urbanas, industriales y pluviales.

En el póster de la Figura 2B, por el contrario, las fotografías son ejemplarizantes y muestran 0 incitan a comportamientos adecuados con el respeto al medio ambiente. Estas acciones se relacionan con el ahorro de agua en nuestras actividades, la reutilización del agua de lluvia, el reciclaje de aceite usado, la reducción del uso de plásticos, o el uso adecuado del inodoro.

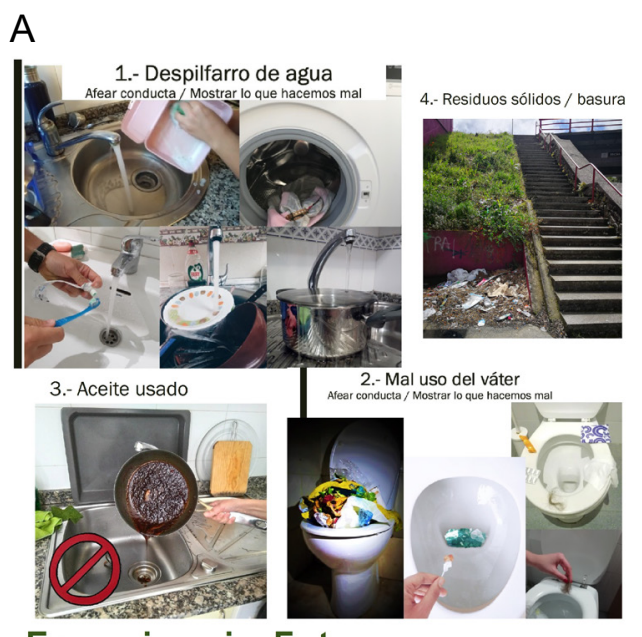

\section{B}

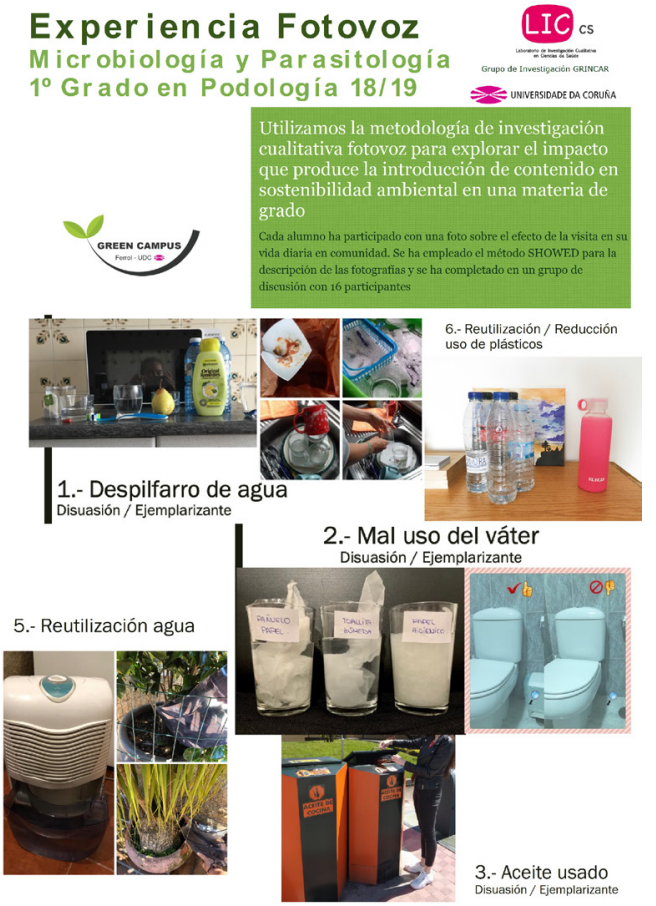

Figura 2: Pósteres realizados con la experiencia fotovoz de alumnos de Podología para el Tablón Green Campus de la Facultad de Enfermería y Podología 
La mayoría de los estudiantes informaron que no habían recibido capacitación en temas de sostenibilidad hasta que llegaron a la Universidad.

Consideraron que la capacitación en sostenibilidad debería comenzar en la infancia en el hogar, más tarde como un contenido transversal en la escuela, y finalmente, debería reforzarse en la Universidad cuando muchos de ellos comienzan sus vidas aparte de su familia.

En sus aportaciones quedó patente que habían aprendido mucho, durante la visita guiada a la planta depuradora, sobre comportamientos ciudadanos nada respetuosos con el medio ambiente y sobre cómo evitarlos. Proclamaron la necesidad de campañas de concienciación públicas en redes sociales o televisión sobre el tema, así como la urgencia sobre la implicación de las empresas en el respeto al medio ambiente.

\section{CONCLUSIÓN}

Diferentes autores han indicado que la metodología fotovoz se puede usar de manera creativa para proporcionar a los estudiantes una actividad agradable que los lleve a la reflexión y al pensamiento crítico sobre sus prácticas en enfermería, psicología o ciencias de la salud (Edwards et al. 2012; Fernandes, Ferreira, \& Marques, 2018; Gallagher \& Stevens, 2015; Leipert \& Anderson, 2012; Lichty 2013).

En nuestra experiencia con estudiantes de podología hemos podido comprobar que esto es cierto por varias evidencias. En primer lugar, por el alto grado de participación en la metodología que se ofertó como voluntaria, tanto a nivel de la realización de las fotografías como en la reflexión en el documento que las describía, y además en la estupenda dinámica desarrollada en la sesión grupal de discusión.

Por otro lado, consideramos muy positiva la utilización de esta metodología cualitativa para complementar actividades formativas que se realizan en entorno real y que implican acciones con consecuencias para la comunidad. Mediante fotovoz se pueden obtener propuestas de mejora para la resolución de problemas que parten de los propios agentes de la comunidad. 
Pensamos que la comunidad universitaria es especialmente rica y participativa para contribuir al éxito de la metodología.

Entre las dificultades de esta metodología podemos señalar que los datos que se generan durante el proceso son muy ricos y con un volumen importante. Eso hace que el análisis cualitativo de los datos es costoso en dedicación y ha conllevado la implicación de cuatro analistas. Estos analistas deben estar previamente formados en investigación cualitativa.

Como fortaleza del método está la posibilidad de generar conocimiento en un tema concreto que puede permitir realizar una publicación científica con los resultados específicos alcanzados.

La utilización de la metodología fotovoz con estudiantes de podología ha generado una oportunidad atractiva de aprendizaje colaborativo respecto a los contenidos microbiológicos, y el despertar y fortalecimiento de la conciencia ciudadana de los estudiantes sobre sostenibilidad medioambiental y el uso responsable del agua.

\section{REFERENCIAS}

Catalani, C. \& Minkler, M. (2010). Photovoice: A review of the Literature in Health and Public Health. Health Education \& Behavior, 37 (3), pp. 424-451.

Edwards, M., Perry, B., Janzen, K., \& Menzies, C. (2012). Using the artistic pedagogical technology of photovoice to promote interaction in the online post-secondary classroom: The students' perspective. Electronic Journal of e-Learning, 10 (1), pp. 3243.

Fernandes C. S., Ferreira, F., \& Marques, G. (2018). The use of the Photovoice methodology to determine the concept of family which nursing students have. Avances en Enfermería, 36 (1), pp. 59-68.

Gallagher, M. R. \& Stevens, C. A. (2015). Adapting and integrating photovoice in a baccalaureate community course to enhance clinical experiential learning. Journal of Nursing Education, 54 (11), pp. 659-662. 
Leipert, B. \& Anderson E. (2012). Rural nursing education: a photovoice perspective. Rural and Remote Health, 12, pp. 2016.

Lichty, L. F. (2013). Photovoice as a pedagogical tool in the community psychology classroom. Journal of Prevention and Intervention in the Community, 41 (2), pp. 89-96.

Wallerstein, N. \& Bernstein, E. (1988). Empowerment education: Freire's ideas adapted to health education. Health Education \& Behavior, 15, pp. 379-394.

Wang, C. C. (2003). Using Photovoice as a participatory assessment and issue selection tool: A case study with the homeless in Ann Arbor. En M. Minkler \& N. Wallerstein (Eds.), Community based participatory research for health (pp. 179-196). San Francisco, CA, US, Jossey-Bass.

Wang, C. C. \& Burris, M. A. (1997). Photovoice: Concept, Methodology and Use for Participatory needs Assessment. Health Education \& Behavior, 24 (3), pp. 369-387. 
